\title{
Mind Wandering and Creativity
}

\section{Stefania Righi*}

Department Neurofarba, University of Florence, Florence, Italy

\section{Introduction}

The term mind-wandering entered the psychological scientific literature about a decade ago, when Smallwood and Schooler [1] defined it as the condition in which everyone, in some situations, found themselves unable to maintain the concentration on the main target, especially when people are involved in tasks of an attentive nature. Specifically, selective attention is the ability to focus attention on information related to a purpose (important for the correct execution of the task), and to keep the attention on such information within a system where there are also strong distractors. This ability to keep the attention focused on a specific objective, and to process only the data related to the task, is necessary for a multitude of daily activities. However, according to Unsworth and McMillan [2], it can happen that the attention needed to carry on an activity has shifted from the relevant information to focus on other stimuli. These can be either external (distractions) or consist of internal thoughts (mind-wandering). Typical examples of mind-wandering are those self-generated thoughts that arise when you are driving, or when you are reading a text: in these situations the attention may have a fluctuating trend and people may be surprised to digress in their mind. These attention spills can lead to unwanted outcomes, such as driving accidents, poor academic performance, or poor / failed performance of a task. Basically it can be said, therefore, that mind-wandering indicates the occurrence of thoughts independent of a stimulus and not related to a specific task, which is commonly associated with poor performance. For example, mind-wandering during reading, is constantly associated with a decrease in the comprehension of the text [3], while the occurrence of mind-wandering during go / no-go tasks is linked to times of reaction (RTs) more variable to stimuli-go and a high error rate in no-go stimuli [4]. Recent research has revealed that mind-wandering is a fundamental part of our daily reflection time (i.e., 20 to $50 \%$ of which an important part is dedicated to planning and preparing for future events) and its frequency is usually high during relatively easy and low difficulty tasks, while decreasing gradually with increasing task difficulty [5]. Currently in the literature there are two different theories that try to explain the relationship between mind-wandering and attentive processes. The first is the so-called theory of perceptual decoupling, which claims that mind-wandering derives from a redirection of attentional resources that are subtracted from the elaboration of the task, to be directed to the maintenance of internal thoughts [6]. According to this line of thought mind-wandering is, therefore, a costly phenomenon because it requires attentive resources. It would be more frequent during tasks with reduced cognitive investment, because more cognitive resources are available for internal thinking. Tasks with a high cognitive load, on the contrary, tend to deplete available resources and therefore make this phenomenon less likely. The alternative theory is the failure of control, according to which mind-wandering does not use attentive resources but, instead, would reflect a temporary decay of attention control processes that should keep the attention of the individual focused on the task [6]. According to this point of view, individuals with low attention control skills are more likely to mind-wandering, because they are less efficient in keeping their attention on a specific task in a continuous way [7]. Following this line of thought, the fact that during difficult tasks the mind-wandering occurs with less frequency, it would be due to the fact that when the cognitive load is high, the subject is required repeated attentive control processes during the completion of the task. Interestingly, when a decoupling process takes place that leads to a dampening in the processing of environmental stimuli the cognitive resources are directed to stimulus-independent thoughts (SIT) [6]. Although disruptive of perception, this decoupling process could have important advantages because it allows the mind to focus in detail on an internal train of thought [8] and so allows the mental consideration of goals other than those in the here and now. Hence, mind-wandering may be considered a state in which the ability to generate and deepen new ideas and goals is improved. Consistently, there are numerous anecdotes of creative ideas occurring to individuals during moments of mind wandering [9]. For example, the creative benefits of incubation intervals are greatest when individuals are occupied by a non-demanding task relative to either a demanding task or no task at all [10]. These considerations have prompted researchers to investigate the relationship between mind wandering and creativity.

There are two views on the mind wandering - creativity relationship. Firstly, mind wandering is associated with creative exploration and expression because it facilitates the formation of novel associations and the recombination of mental images. This can be a source of creative ideas [11-13] because during mind wandering a person's imagination is relatively undisturbed by stimulation from the environment. Secondly, the other view regarding the mind wandering - creativity relationship, suggest that mind wandering could be negatively related to creativity because the performance on divergent and convergent creative tasks are impaired by the lack of focus during mind wandering [14]. Remarkably, the mind wandering - creativity relationship may depend on the cognitive load required by the task. Considering that the mind wandering is more frequent in non-demanding relative to demanding tasks [15], the stimulus-independent thoughts, perhaps particularly when it occurs while engaging in an easy task, can contribute to the creative benefit of an incubation interval. However, according to the first theory, the literature provides us with a series of studies that provide indirect evidence that mind-wandering may improve creativity. This creativity enhancement may be modulated by a change in the breadth of attentional allocation and in particular by loosening the reins on inhibitory control that individuals with attention deficit hyperactivity disorder (ADHD), notoriously associated with greater mind-wandering, tend to have higher scores in creativity tests and to be more creative in their daily life, compared to individuals without

*Corresponding author: Stefania Righi, Department Neurofarba, University of Florence, Florence, Italy, Tel: + 055 2755051; E-mail: stefania.righi@unifi.it

Received: May 04, 2018; Accepted: May 09, 2018; Published: May 16, 2018

Citation: Righi S (2018) Mind Wandering and Creativity. Clin Exp Psychol 4: 193 doi: $10.4172 / 2471-2701.1000193$

Copyright: () 2018 Righi S. This is an open-access article distributed under the terms of the Creative Commons Attribution License, which permits unrestricted use, distribution, and reproduction in any medium, provided the original author and source are credited. 
ADHD. Another study has instead found that individuals with a reduced latent inhibition capacity (defined as the ability to examine previously considered content irrelevant from conscious awareness) tend to have higher scores in creative tasks and to report new and useful ideas, as well as creative results, in everyday life [16]. It has also been found that there are particularly creative individuals who tend to be easily distracted by irrelevant or peripheral stimuli, and therefore have difficulty maintaining focus on the focus. These results, although indirect, suggest that a cognitive style characterized by mindwandering would seem to favor creativity. There are also direct evidence in favor of the hypothesis according to which mind-wandering has a beneficial effect on creativity. In particular, we can refer to the study by Baird et al. [17] who investigated the effects of mind-wandering, both dispositional and situational, with respect to creativity. To evaluate the latter, the subjects of the study were invited to generate original ideas, related to the unusual use of objects, between one task and the other the participants were interrupted for 12 minutes (which serve as an incubation interval for the creativity). The researchers found that the most creative results were obtained as a result of less demanding tasks, which allowed subjects to spend 12 minutes between tasks to mindwandering. This indicates that mind-wandering can be fostered by a series of situational factors that can, therefore, influence creativity.

\section{Conclusion}

These promising results suggest the need to deepen the relationship between mind wandering and creativity with further studies that may lead to both a better understanding of the ways in which new ideas are generate in mind wandering state and practical proposal for improving creativity [18].

\section{References}

1. Smallwood J, Schooler JW (2006) The restless mind. Psychol Bull 132: 946958.

2. Unsworth N, McMillan BD (2014) Similarities and differences between mindwandering and external distraction: A latent variable analysis of lapses of attention and their relation to cognitive abilities. Acta Psychol 150: 14-25.

3. McVay JC, Kane MJ (2012a) Why does working memory capacity predict variation in reading comprehension? On the influence of mind wandering and executive attention. J Exp Psychol Gen 141: 302-320.
4. Stawarczyk D, Majerus S, Maj M, Van der Linden M, D’Argembeau A (2011) Mind-wandering: Phenomenology and function as assessed with a nove experience sampling method. Acta Psychol 136: 370-381.

5. Song X, Wang X (2012) Mind wandering in Chinese daily lives-An experience sampling study. PLoS ONE 7: e44423.

6. Schooler JW, Smallwood J, Christoff K, Handy TC, Reichle ED, et al. (2011) Meta-awareness, perceptual decoupling and the wandering mind. Trends Cogn Sci 15: 319-326.

7. McVay JC, Kane MJ (2012b) Drifting from slow to "d'oh!": Working memory capac- ity and mind wandering predict extreme reaction times and executive control errors. J Exp Psychol Learn Mem Cogn 38: 525-549.

8. Frith $C D$, Frith $\cup(2006)$ The neural basis of mentalizing. Neuron 50: 531-534.

9. Poincaré H (1952) Mathematical discovery.

10. Sio U, Ormerod TC (2009) Does incubation enhance problem solving? A metaanalytic review. Psychol Bull 135: 94-120.

11. Shepard RN (1978) The mental image. Am Psychol 33: 125-137.

12. Flowers JH, Garbin CP (1989) Creativity and perception. In: Glover JA Ronning RR, Reynolds CR (eds.). Handbook of Creativity. Plenum Press, New York. pp: 147-162.

13. Zedelius CM, Schooler JW (2016) The richness of inner experience: relating styles of daydreaming to creative processes. Front Psychol 6: 2063.

14. Hao N, Wu M, Runco MA, Pina J (2015) More mind wandering, fewer original ideas: Be not distracted during creative idea generation. Acta Psychol 161: 110 116.

15. Smallwood J (2009) When is your head at? An exploration of the factors associated with the temporal focus of the wandering mind. Conscious Cogn 18: $118-125$.

16. Fink A, Slamar-HalbedI M, Unterrainer HF, Weiss EM (2012) Creativity: Genius, madness, or a combination of both?. Psychol Aesthet Creat Arts 6: 11-18.

17. Baird B, Smallwood J, Mrazek MD, Kam JWY, Franklin MS, et al. (2012) Inspired by distraction: mind wandering facilitates creative incubation. Psychol Sci 23: 1117-1122.

18. Smallwood J, Nind L, O'Connor RC (2009) When is your head at? An exploration of the factors associated with the temporal focus of the wandering mind. Conscious Cogn 18: 118-125. 